Gens de Nouméa, gens des îles, gens d'ailleurs... Langues et identités en Nouvelle-Calédonie de Sophie Barnèche

\title{
Benoît Carteron
}

\section{OpenEdition}

12 Journals

\section{Édition électronique}

URL : http://journals.openedition.org/jso/2432

DOI : $10.4000 /$ jso.2432

ISSN : 1760-7256

\section{Éditeur}

Société des océanistes

\section{Édition imprimée}

Date de publication : 15 décembre 2008

Pagination : 361-363

ISBN : 978-2-85430-012-3

ISSN : 0300-953x

\section{Référence électronique}

Benoît Carteron, « Gens de Nouméa, gens des îles, gens d'ailleurs... Langues et identités en NouvelleCalédonie de Sophie Barnèche », Journal de la Société des Océanistes [En ligne], 126-127 | Année 2008, mis en ligne le 01 décembre 2008, consulté le 23 septembre 2020. URL : http:// journals.openedition.org/jso/2432 ; DOI : https://doi.org/10.4000/jso.2432

Ce document a été généré automatiquement le 23 septembre 2020.

(c) Tous droits réservés 


\title{
Gens de Nouméa, gens des îles, gens d'ailleurs... Langues et identités en Nouvelle-Calédonie de Sophie Barnèche
}

\author{
Benoît Carteron
}

\section{RÉFÉRENCE}

Sophie Barnèche, 2005. Gens de Nouméa, gens des îles, gens d'ailleurs... Langues et identités en Nouvelle-Calédonie, Paris, L'Harmattan, coll. Espaces discursifs, 327 p.

1 Cet ouvrage est la publication de la thèse de doctorat de $3^{\mathrm{e}}$ cycle, soutenue en 2004 (université de Rouen), dont le titre, «L'identité linguistique et culturelle des jeunes de Nouméa. Une étude des pratiques langagières dans la cité de Riverstar (Rivière-Salée) », décrit de façon plus explicite son contenu. Sophie Barnèche examine ici le dilemme qui se pose aux jeunes Océaniens quant à l'usage des langues vernaculaires, «langues identitaires", face au français, "langue d'intégration». Ce conflit linguistique est particulièrement mis en évidence, à Nouméa, en raison du caractère plurilingue et pluriethnique de la ville. Et ces enjeux de compétition linguistique sont principalement ceux de la communication quotidienne et de l'intégration économique à la société dominante.

2 En amont de l'introduction des langues vernaculaires dans l'enseignement ${ }^{1}$, l'auteur propose « d'étudier en profondeur la réalité et l'évolution des pratiques linguistiques à Nouméa » (p.10). Pour cela, même si les jeunes sont au centre de son étude, l'auteur s'intéresse en premier lieu à leurs parents, afin d'examiner les pratiques de transmission du patrimoine linguistique, se donnant pour objectif d'établir «quelles sont les stratégies linguistico-identitaires qu'ils mettent en œuvre au quotidien plus ou moins consciemment?» (ibid.). En second lieu, elle appréhende les représentations et 
les attitudes des jeunes de la cité concernant les langues qui les entourent, afin de comprendre l'évolution des pratiques linguistiques, mais aussi et surtout «les revendications identitaires de ces jeunes Océaniens qui grandissent en ville» (p. 11). En cela réside la véritable richesse de cette étude: les pratiques linguistiques y sont considérées comme analyseur de l'intégration des jeunes à la vie urbaine, permettant d'éclairer la manière dont se structure l'appartenance sociale et se construit l'identité individuelle parmi les collectifs en présence: catégorisations ethniques, quartier, société urbaine dominante, tribus dont sont originaires les jeunes.

3 L'ouvrage est divisé en trois parties. Dans la première, Sophie Barnèche développe les orientations théoriques et les choix méthodologiques de son étude. Dans la seconde et la troisième partie, elle approfondit les deux axes thématiques et problématiques de la recherche : l'usage et la transmission des langues vernaculaires dans la cité étudiée, les pratiques langagières dans leurs rapports avec l'affirmation identitaire des jeunes Océaniens. Il faut noter que les jeunes Océaniens dont il est question sont en très grande majorité des Kanak, principalement issus des îles Loyauté. La cité de 600 habitants dans laquelle ils vivent constitue un sous-ensemble du quartier périphérique de Rivière Salée.

4 L'enquête par entretiens, principale source d'analyse, a concerné quatorze jeunes de seize à vingt-cinq ans et huit adultes parmi leurs parents. Sophie Barnèche a pu légitimer sa présence auprès des habitants grâce à une mission d'étude confiée par la mairie de Nouméa avec la réhabilitation du quartier, d'où son acceptation par la population facilitée aussi par l'obtention d'un logement dans cette cité où elle a résidé plus d'un an. Cette observation participante lui a, en outre, permis de mettre en relief le décalage entre les pratiques déclarées et les conduites observées. Enfin, l'enquête a été complétée par un questionnaire rempli par cent quarante et une personnes de la cité. La «recherche impliquée» (p.19) que justifie Sophie Barnèche s'est non seulement traduite par le lien entre la participation à l'action sociale urbaine et la recherche approfondie, mais aussi par la capacité de l'auteur, particulièrement appréciable, à prendre la mesure des effets de son statut d'enquêtrice «blanche » et de déceler ainsi la part de réaction à la situation d'enquête dans les propos des jeunes.

5 La thématique des langues et de l'identité fait l'objet d'une double problématisation. L'une, sociolinguistique, considère les pratiques langagières comme révélatrices et constitutives des conflits et déséquilibres entre les groupes. Les usages de la langue chez les jeunes sont ainsi à apprécier ici vis-à-vis du français légitime de la société dominante. Le second axe problématique se précise avec l'apport de la psychologie interculturelle. La construction identitaire y est abordée sous son angle dynamique, comme autant de réponses provisoires et renouvelées auxquelles donnent lieu les rapports de l'individu avec son environnement. En situation de contacts et conflits culturels, la lecture en termes de stratégies identitaires éclaire singulièrement la façon dont les individus se démêlent des obstacles rencontrés et des situations dévalorisantes pour maintenir ou restaurer une valeur et une cohérence à leur être.

6 La construction identitaire des jeunes est placée dans son contexte urbain et l'enjeu de l'intégration socioéconomique à la ville. Rapports aux langues et à l'espace se croisent dans la résistance qu'opposent les jeunes à la double dépréciation dont ils sont l'objet : relégués comme habitants de quartiers à majorité océanienne défavorisée (en même temps que dévalorisés par leur usage non standard du français) et stigmatisés par ceux de leur communauté d'origine comme des "gens de Nouméa " (en même temps que 
culpabilisés pour leur maitrise imparfaite de leur langue d'origine). Dès lors, l'espace du quartier et de la cité est survalorisé par une revendication d'appartenance à une "communauté de vécu», constituée par les pairs du quartier, ce qui permet de se reconstruire une identité positive et cohérente. Dans cet espace s'affirme "en même temps leur identité ethnique, perçue comme motif de rejet de la part de la société dominante, et leur identité "urbaine", objet d'une stigmatisation de la part de la communauté d'origine » (p. 69).

7 Les langues d'origine se transmettent difficilement ou pas du tout en milieu urbain. Ceux qui sont nés ou arrivés jeunes en ville font un usage spontané du français, tandis que la pratique de la langue vernaculaire est restreinte au cercle familial. Les parents interrogés se montrent relativement passifs dans la transmission et indifférents face au risque de disparition des langues. Cette attitude s'explique en bonne partie par la certitude que les langues seront toujours parlées à la tribu, considérée comme le lieu véritable de la conservation de la langue et de la culture. En ville, le français s'impose, justifié par l'idéologie qui confère au français le statut unique de langue de communication et d'accès au savoir; ce qui se traduit par une "préoccupation pragmatique » (p.164) des parents: l'intégration à la société dominante. L'accès à l'emploi et la réussite scolaire des enfants, pour lesquels le français est nécessaire, sont jugés plus importants que la préservation de la culture d'origine. La stratégie d'assimilation n'en demeure pas moins essentiellement économique et d'accès au mieux-être matériel. Se développe ainsi une "culture océanienne urbaine hybride " (ibid.), fière de son identité mais recherchant le mieux-être matériel. Dans ce contexte, les langues vernaculaires sont renvoyées au «statut noble mais fatal de pur symbole identitaire » (p. 159).

8 L'attachement à la langue d'origine est plus fortement exprimé chez les jeunes. Assimilée aux racines, perçue comme ce qui fait le lien et permet la communication avec les gens de la tribu, notamment les vieux, la langue permet aux jeunes de se sentir participer affectivement à la culture et sa transmission. Mais autant la portée symbolique des langues d'origine s'accentue chez les jeunes, autant leur maitrise est faible. Il en résulte une «culpabilisation linguistique» (p.181) et une insécurité dans l'usage de la langue, que conforte l'attitude stigmatisante des gens de la tribu. La perte de la langue d'origine est synonyme d'une souffrance affective et un malaise identitaire chez les jeunes. Perte qui selon l'auteur « pourrait jouer un rôle décisif à la fois dans la rupture avec le milieu tribal et dans la rupture avec la société dominante» (p. 187). Dans ce contexte, le «français kaya " ou «français à nous» devient la langue du quartier. Il conditionne l'appartenance au groupe de pairs, fonctionne comme une contre norme face à l'échec de l'apprentissage scolaire et permet de s'exprimer librement par l'adaptation et le mélange. La stigmatisation de leur français, déformé et défectueux vis-à-vis de la norme, est ainsi retourné en "valeurs positives de décontraction, de liberté et de créativité » (p.47) par un usage non conforme de la langue devenant pour les jeunes un emblème identitaire.

9 Dans les stratégies mises en œuvre, la revendication linguistique s'associe à la revendication ethnique, particulièrement saillante dans les discours. Les locuteurs des langues vernaculaires ont intériorisé la dépréciation de leur langue face à l'idéologie unilinguiste unilingue? française. Il en ressort une ambivalence profonde des jeunes face à la langue légitime : acceptation de son prestige du français et résistance dans les rapports entretenus avec elle. L'opposition du «parler phrasé », renvoyant au monde 
des «eux ", au "parler simple» du monde des «nous » est révélatrice des barrières socioethniques et du sentiment d'exclusion économique des jeunes. Parler le français standard revient à "afficher une volonté de s'intégrer pleinement au "monde des Blancs" et pour beaucoup cela équivaut à renier son identité d'Océanien » (p. 231). Les attitudes et représentations des jeunes se situent sur un continuum dont les deux pôles idéologiques sont l'intégration à la société occidentale à une extrémité et la revendication kanak à l'autre. L'ambivalence des jeunes se traduit par un rapport fluctuant entre ces idéologies et la plupart sont tiraillés entre deux représentations divergentes :

« l'une rejetant la langue française comme l'outil et le symbole de l'oppression des

Blancs; l'autre l'acceptant comme sienne et la percevant comme une moyen de communication nécessaire et bénéfique. » (p. 248)

10 Alors que les gens de la tribu jugent négativement ces jeunes urbains, l'antagonisme ville/tribu renvoie à une double assignation : s'assimiler au Blanc ou rester Kanak. Face à cela, l'auteur identifie deux stratégies identitaires pour se construire malgré tout une image de soi positive chez les jeunes : une identité « réactionnelle » par survalorisation de l'appartenance ethnique, rejet de la société dominante et des différents groupes sociaux confondus avec des catégories ethniques globalisantes; une identité "océanienne urbaine» qui consiste à retourner la stigmatisation, c'est-à-dire revendiquer son origine ethnique tout en la renvoyant au passé et à s'identifier au quartier en louant l'évolution que représentent les modes de vie urbains. Chez la majorité des jeunes rencontrés, l'identité réactionnelle marque le pas face à la préoccupation pragmatique. En dépit d'un profond attachement à leurs racines, les jeunes mettent la tribu à distance et affirment un attrait pour la ville, associée à la modernité et au progrès. Ils s'affichent en rupture face à une appartenance kanak uniformisante pour valoriser "une identité commune basée sur des similitudes culturelles et des traditions partagées » (p. 262). L'identité océanienne urbaine permet ainsi de revendiquer les origines, vanter le métissage, démontrer l'appropriation de la ville, valoriser le mélange des langues, s'opposer aux valeurs dominantes comme se détacher de valeurs traditionnelles. L'ancrage urbain se double cependant d'une "emblématisation de l'ethnicité »(p.292): la revendication de l'origine culturelle s'exacerbe d'autant plus qu'elle se vide de son contenu réel et que les jeunes s'en sentent dépossédés.

11 On regrettera quelques lacunes : une présentation trop sommaire de la cité et l'absence d'indication sur les dates de réalisation de la recherche sur le terrain (même si on devine qu'il s'agit du début des années 2000). Sur le plan formel, une longue première partie théorique, quoique éclairante, amène l'auteur à se répéter dans les développements ultérieurs. Au final, il s'agit cependant d'un ouvrage très riche dont le foisonnement est le fruit d'une analyse fouillée des discours produits, sans jamais perdre de vue le contexte global et la situation de chaque personne interrogée. La complexité de la position d'entre deux des jeunes Kanak vivant en ville et le traitement de leur spécificité culturelle sont particulièrement bien rendus, la comparaison avec les situations d'immigrations internationales en est d'autant plus pertinente. Pour l'enseignement des langues vernaculaires, auquel Sophie Barnèche consacre une partie de sa conclusion, cet ouvrage est un appui précieux pour en comprendre les obstacles et envisager les conditions d'une véritable transmission de la langue d'origine. 


\section{NOTES}

1. L'enseignement des langues kanak dans le primaire a été officialisé à la rentrée 2006.

\section{AUTEURS}

BENOÎT CARTERON

CERIPSA-CARTA, université catholique de l'Ouest, Angers 